Espac. Saúde. 2021;22:e774

Doi 10.22421/1517-7130/es.2021v22.e774

(C) 2018 - ISSN 15177130

\title{
ENSAIO
}

\section{Curricularização da extensão universitária em saúde: uma proposta com uso do diagnóstico comunitário}

\author{
Curricularization of university extension health courses: a proposal with the \\ use of community diagnosis \\ "Curriculización" de la ampliación universitaria en salud: una propuesta que \\ utiliza el diagnóstico comunitario
}

Leandro Rozin ${ }^{1}$, Luiza Tatiana Forte ${ }^{2}$

\begin{abstract}
${ }^{1}$ Enfermeiro. Mestre em Biotecnologia Aplicada à Saúde da Criança e do Adolescente pela Faculdades Pequeno Príncipe. Coordenador do Programa de Extensão Social e Comunitário (PESC) na Faculdades Pequeno Príncipe. Curitiba, Paraná.

${ }^{2}$ Psicóloga. Mestre em Educação pela Pontifícia Universidade Católica do Paraná. Diretora de Extensão na Faculdades Pequeno Príncipe. Curitiba, Paraná.
\end{abstract}

\section{INTRODUÇÃO}

A partir do movimento de reforma sanitária no Brasil, a demanda pela formação profissional baseada nas necessidades de saúde da população tem aumentado e tornou-se foco relevante na atualidade. A reorganização dos currículos dos cursos de graduação na área da saúde tem potencializado o redimensionamento do ensino à integração do serviço e comunidade para atuação eficiente no Sistema Único de Saúde, principalmente no campo da Atenção Primária em Saúde (APS) ${ }^{1}$.

O anseio volta-se ao campo social da saúde para integração do ensino com o serviço, a fim de contribuir para melhoria da situação de saúde das comunidades durante a formação e desenvolvimento 
dos futuros profissionais. As universidades vêm se concentrando para o alcance das competências, sendo necessário romper o modelo tradicional segmentado, voltando-se à coerência do processo saúde-doença, aos determinantes de risco e proteção à saúde, tornando o cuidado voltado para a atenção primária de forma integral ${ }^{2-3}$.

A integração universidade serviço e comunidade requer compromisso social com a sistematização de fazeres, com o sistema de saúde do País e com o desenvolvimento profissional no contexto de seu impacto e resolubilidade em problemas de saúde. Para o alcance desses objetivos, torna-se indispensável a articulação de saberes da saúde coletiva, epidemiologia, políticas de saúde, da gestão e das ciências sociais, atrelados à realidade das comunidades. Destaca-se que as práticas de saúde devem ser entendidas como respostas sociais aos problemas e necessidades de saúde da coletividade ${ }^{4-5}$.

Os eixos norteadores das Diretrizes Curriculares Nacionais (DCN) dos cursos da área de saúde são determinados no sentido de se conectar com a realidade do Sistema Único de Saúde, destacando a formação dos saberes. Para o alcance das competências, cada eixo contempla o que se deve saber cognitivamente para obter o conhecimento, o que deve aprender para realizar as atividades profissionais práticas a fim de obter habilidades, e o que deve contribuir com a formação do ser humano, para formação ética, cidadã e humanitária, caracterizada pelas atitudes profissionais ${ }^{3,6}$.

Outro aspecto importante destacado é o foco em conteúdos fundamentais dos cursos de graduação das áreas de saúde, articulando o processo saúdedoença do cidadão, da família e da comunidade com a realidade epidemiológica e profissional, proporcionando a integralidade das ações do cuidado em saúde. Essa articulação vislumbra a formação de melhores profissionais para atuarem na complexidade da realidade social, política e cultural, que demanda um desafio no ensino superior ${ }^{2-3}$.

Os docentes são fundamentais para a qualificação do ensino, no desenvolvimento de metodologias e avaliações inovadoras, desafiadoras, inteligentes, criativas e estimuladoras que promovam interações com a comunidade e os serviços a fim de qualificar formação real do exercício da profissão $0^{1,3,7}$.

Nesse viés, de forma contributiva e complementar às DCNs, o Ministério da Educação, por meio do Conselho Nacional de Educação e Câmara de Educação Superior estabeleceu por meio da Resolução nº 7 de 18 de dezembro de 2018, as Diretrizes para a Extensão na Educação Superior Brasileira, conceitualmente definida como "atividade que se integra à matriz curricular e à organização da pesquisa, constituindo-se em processo interdisciplinar, político educacional, cultural, científico, tecnológico, que promove a interação transformadora entre as instituições de ensino superior e os outros setores da sociedade" 8 .

A Resolução determina que $10 \%$ do total da carga horária curricular dos cursos de graduação devem ser cumpridas com atividades de extensão, voltadas à comunidade externa, em que as atividades/ações de extensão deverão fazer parte da matriz curricular dos cursos, desenvolvidas por meio das modalidades de programas, projetos, cursos e oficinas, eventos e/ou prestação de serviços ${ }^{8}$.

As atividades de extensão são expressas como práticas diferenciadas em meio à sociedade. Contribuem, nas dimensões profissional e pesquisador, para a formação integral do estudante-cidadão ético, crítico e responsável, negociador, construtivo e transformador, respeitando e promovendo a interculturalidade?

São desenvolvidas por meio de iniciativas e ações que expressem o compromisso social, em consonância com as políticas ligadas às diretrizes para educação ambiental, educação étnico-racial, direitos humanos e educação indígena, com incentivo à atuação da comunidade acadêmica e técnica no enfrentamento das questões reais, prevalentes na sociedade. Contribui, ainda, para a produção e a construção de conhecimento atualizado e coerente, voltado para o 
desenvolvimento social, equitativo, sustentável e em consonância com a realidade?

Com a necessidade desse olhar sobre o paradigma determinista da extensão universitária, fundamental para mudanças na formação dos profissionais de saúde, as Instituições de Ensino Superior (IES) requerem investimentos em metodologias que promovam a movimentação de pensamento acadêmico a partir das realidades, aproximação com os sujeitos sociais e com os serviços de saúde.

Movimentar o pensamento é a base para a aprendizagem significativa, resulta da observação da realidade aparente, que por meio de análises e reflexões almejam o concreto, o que há de essencial no objeto originado a partir das múltiplas determinações. Complementarmente, o pensamento complexo visa mover, articular os diversos saberes compartimentados nos variados campos de conhecimento, sem perder a essência e a particularidade. Se constitui como requisito para o exercício da interdisciplinaridade, almeja o conhecimento multidimensional, estando, no entanto, ciente de que o conhecimento completo é inatingível e indica que o caminho é dialógico e a realidade é alterada constantemente ${ }^{10-11}$.

Nesse sentido, o Diagnóstico Comunitário (DC) pode contribuir no processo de ensino e aprendizagem por ser uma ferramenta sistematizada, articulada ao contexto interdisciplinar e multidimensional. Proporciona aprendizagem integral de profissionais de saúde pautada em reflexões complexas diante da singularidade dos saberes identificados na realidade vivenciada em comunidades cobertas por equipes de saúde.

O DC articula saberes da saúde coletiva que permitem o alcance dos objetivos propostos nas DCNs apontadas nas competências, embasam as atividades de extensão determinadas pelas necessidades reais da sociedade e consolidam a integração ensino, serviço e comunidade que contemplam os eixos necessários para a formação de profissionais de saúde ${ }^{12}$.

Proposto por Dominguz em 1998, estruturado como método por Péchevis em 2000 e disponibilizado como instrumento indispensável para atuação eficaz na atenção primária à saúde pelo Ministério da Saúde, o DC objetiva identificar problemas, necessidades e recursos nas comunidades. Representa a ferramenta necessária para organizar o processo de trabalho de equipes multiprofissionais de saúde com base descentralizada, para o enfrentamento de problemas heterogêneos e necessidades distintas diante dos perfis de vulnerabilidade social ${ }^{12-16}$.

Com o conhecimento da realidade, dos problemas de saúde prevalentes e seus riscos, das necessidades intersetoriais que interferem no processo saúdedoença e dos recursos disponíveis em meio à comunidade, o planejamento em saúde torna as intervenções resolutivas, necessárias e relevantes. Realizado por meio da coleta sistematizada de informações, com dados mistos, é possível conhecer os indicadores de saúde, determinantes de risco e recursos favoráveis que dão base ao planejamento de ações para qualificação da saúde em nível local e permite avaliar permanentemente a situação de saúde das comunidades ${ }^{12-16}$.

Portanto, o emprego do DC na formação de profissionais de saúde cumpre as determinações das DCNs, aproxima e efetiva a articulação entre o ensino e aprendizagem baseada em problemas reais, incorpora a pesquisa por tratar-se de uma metodologia de coleta e análise sistêmica de dados na busca de novos conhecimentos e favorece a extensão, desenvolvida por meio de ações que expressam o compromisso social da academia. Logo, cumpre o dever da universidade em ocupar-se de ações sociais do ponto de vista da promoção da saúde pautadas em determinantes das condições de vida dos diversos grupos populacionais como ponto de partida para a realização de intervenções necessárias à sociedade que impacta na melhoria dos níveis de saúde e o controle de doenças ${ }^{3,6}$.

A articulação desses saberes que garantem a formação qualificada de profissionais de saúde, na vertente contemporânea desafiadora, necessita sustentação 
de um paradigma que permita a reestruturação do pensamento e de fazeres, exige originalidade e ousadia, pois corrobora com as necessidades de superação de modelo de ensino tradicional e fragmentado para a integralidade do conhecimento na prática profissional embasado nas demandas reais da sociedade.

Dessa forma, a presente discussão é resultante de um ensaio teórico reflexivo, cuja finalidade é recomendar o Diagnóstico Comunitário como ferramenta sistematizada para o desenvolvimento das ações e atividades de extensão curricular na formação universitária em saúde, a partir da conjuntura ensino, serviço e comunidade.

\section{MÉTODO PARA REALIZAÇÃO DO DIAGNÓSTICO COMUNITÁRIO}

No ensino das ciências da saúde, o diagnóstico da comunidade teve destaque na década de 1980, como adaptação do Método de Projeto. Sistematizado para o reconhecimento da área de abrangência comunitária compreende consecutivamente a delimitação dos objetivos e metas, elaboração dos instrumentos e coleta de dados, apuração e interpretação dos dados através de conceitos, tabelas e gráficos, análise e discussão dos resultados com as lideranças da comunidade. Diante da situação identificada, segue a proposição de soluções e elaboração do documento final que constitui a avaliação diagnóstica da área trabalhada ${ }^{17}$.

$\mathrm{O}$ método direcionado à construção do $\mathrm{DC}^{12}$, originalmente disponível para as equipes de saúde da atenção primária, segue etapas semelhantes ao desenvolvido em estratégias de ensino e viabiliza as ações de extensão universitária, propondo sistematicamente sete etapas que dão base ao conhecimento da realidade e possibilita as ações de intervenção em saúde de forma consciente e planejada (figura 1).

Figura 1 - Espiral para definir as ações de extensão a partir do Diagnóstico Comunitário.

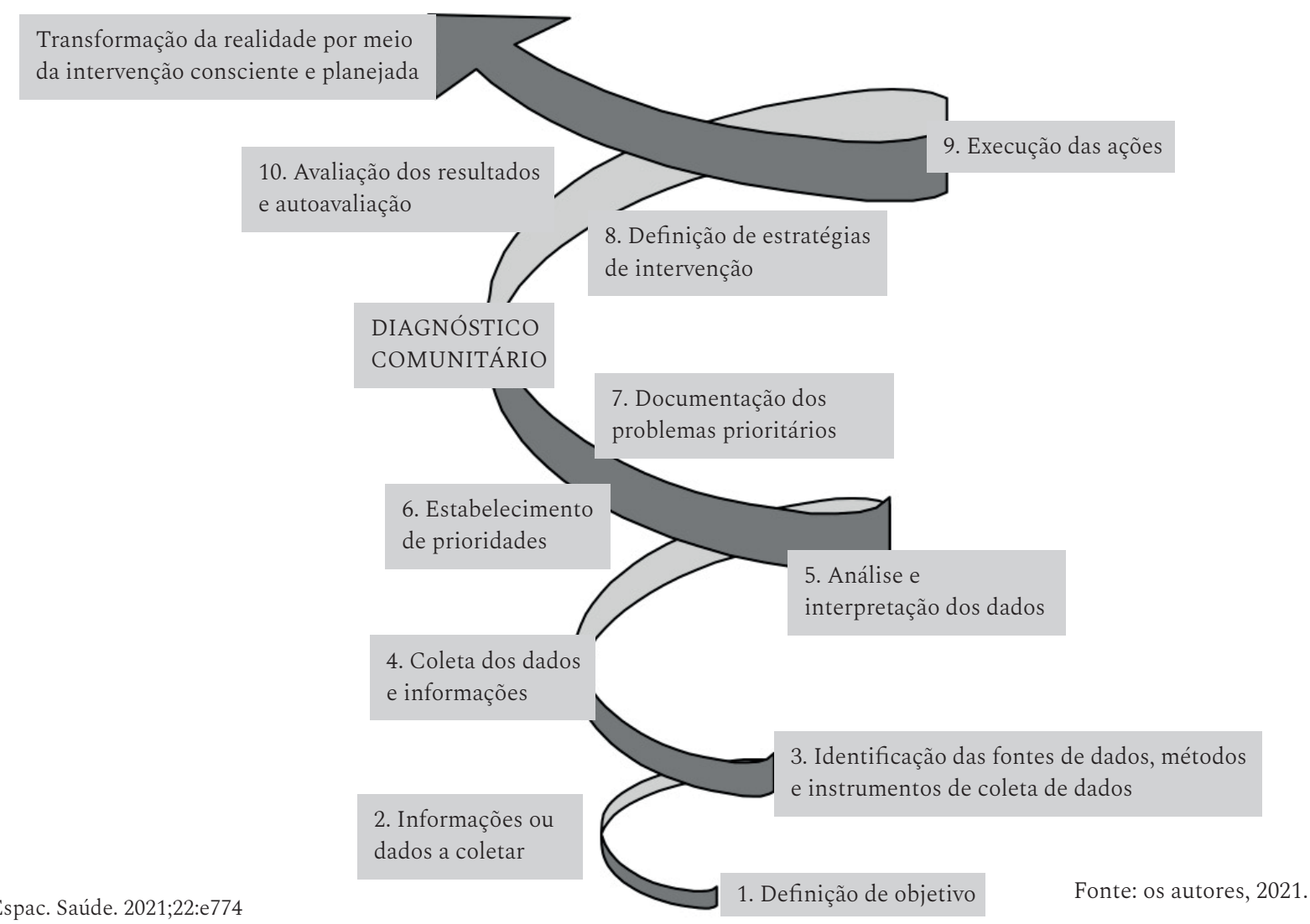




\section{Definição de objetivo}

Nessa etapa, é definida a finalidade que se propõe o DC, de forma ampla e geral para identificar problemas, necessidades e recursos, ou de forma específica direcionado a grupos ou agravos à saúde pontuais de um território, quando se pretende levantar informações e sobre um determinado problema de saúde, comportamento, determinantes, cobertura assistencial, grupos vulneráveis, entre outros, principalmente quando não existem dados preexistentes claros e suficientes. Para efetivar as ações de extensão curricular, os objetivos de aprendizagem da disciplina ou módulo devem estar contemplados no objetivo do DC.

\section{Informações ou dados a coletar}

Nessa fase, devem-se determinar as informações necessárias que possibilitem alcançar os objetivos propostos. Como exemplo, as características da comunidade com o contexto histórico, clima, vegetação, meio ambiente, zona rural e urbana, habitação, escolas, meios de comunicação, transporte, áreas de mata, loteamento, coleta de resíduos, tipo de vias para circulação.

Informações sobre a demografia e indicadores de saúde: número de habitantes e estrutura por idade, sexo, natalidade, mortalidade, morbidade, cobertura vacinal. A situação socioeconômica e cultural: atividades realizadas na comunidade e recursos locais, situação de emprego, categorias profissionais, salários, escolaridade, custo de vida, organização familiar, tipos de religião, atividades culturais, educação, lazer.

A organização administrativa sanitária: legislação sanitária, prioridades nos planos de saúde do município, redes de atenção, estrutura e equipamentos existentes, atividades de saúde existentes, acessos aos serviços de saúde, profissionais de saúde, práticas integrativas e complementares, entre outros.

\section{Identificação das fontes de dados, métodos e instrumentos de coleta de dados}

É a fase em que ocorre a definição da fonte das informações que contemplam dados preexistentes ou a coletar. A escolha de métodos que oportunizam a identificação, por meio de instrumentos de coleta, dados quantitativos e qualitativos que resultem em informações necessárias. Distinguem-se os dados preexistentes disponíveis nas fontes que possuam informações sociodemográficas, de vigilância em saúde com dados epidemiológicos disponíveis nos Sistemas de Informação em Saúde (SIS), ambientais e sanitários, nas fichas E-SUS - Atenção básica como no Sistema de Informação em Saúde para a Atenção Básica (SISAB), além de outros registros do serviço de saúde.

Para os dados não registrados em fontes, sugere-se a criação de formulários ou instrumentos estruturados ou semiestruturados, validados ou não em outras pesquisas. A metodologia que mais se aproxima nessa fase é a descritiva, do tipo levantamento, quando houver fonte de dados secundários, ou inquéritos de saúde quando não houver dados prévios. Porém, a abordagem mista torna-se relevante para a totalidade dos dados, a depender do objetivo da realização do DC.

\section{Coleta dos dados e informações}

Nessa fase, ocorre a coleta dos dados e informações a partir dos instrumentos predefinidos nas fontes preexistentes ou a serem coletados. Para dados preexistentes de indicadores de saúde, sociodemográficos, situação sanitária, ambiental, a busca ocorrerá na fonte disponível oriunda da vigilância em saúde, sistemas de informação, relatórios e outros registros de gestão.

Para os dados e informações a serem coletados, é fundamental a participação e contato com a comunidade por meio de lideranças comunitárias ou conselho local de saúde, equipes e gestores do 
serviço de saúde. Os dados e informações também podem ser coletados em visitas domiciliares ou ao território, a depender do tempo disponível e objetivo do DC. Os profissionais de saúde e gestor do serviço têm papel fundamental na aproximação aos dados e informações, desatacando-se o Agente Comunitário de Saúde (ACS) como articulador e conhecedor do território que facilita a inserção na comunidade.

\section{Análise e interpretação dos dados}

Sugere-se a categorização dos problemas, necessidades e recursos identificados a partir dos dados mistos analisados. Calculam-se e julgamse os indicadores e taxas prevalentes de atenção, as vulnerabilidades passíveis de monitoramento e intervenção, fatores que desempenham papel importante na saúde da comunidade, canais e locais de comunicação, grupos e áreas de risco e opinião da comunidade e o que julgam prioridades nos agravos ou necessidades de saúde.

A análise dos determinantes da saúde pode ser agrupada em categorias distintas que retratam o processo saúde-doença, abrange os comportamentos pessoais e estilo de vida, fatores sociais, condições de vida e de trabalho, acesso e busca de serviços de saúde, condições gerais socioeconômicas, culturais e ambientais, além de fatores intrínsecos como predisposição genética a partir do uso de genograma, faixa etária, sexo e raça. Esse é o momento em que se pode definir a delimitação da área de atenção a partir do mapeamento do território e microáreas definidas conforme os indicadores identificados, riscos sociais e barreiras territoriais.

\section{Estabelecimento de prioridades}

Abaliza os problemas urgentes passíveis de intervenção identificados na análise. Após o julgamento dos dados e informações, devem-se estabelecer prioridades articuladas à percepção da equipe de saúde, comunidade e gestor, pactuando conjuntamente as prioridades de ação para o território.

As prioridades originadas desse consenso serão foco e darão base ao planejamento de ações de saúde para melhoria das condições e indicadores. Os recursos disponíveis no território são fatores essenciais na programação das ações.

\section{Documentação dos problemas prioritários}

Registro que define com precisão os problemas resultantes da apreciação e discussão com a comunidade, gestor e profissionais de saúde. Essa fase oportuniza prever as causas determinantes da situação de saúde na comunidade, os fatores de risco e identifica os recursos que poderão ser utilizados para proposta de intervenção.

A documentação do processo tem por objetivo formalizar o DC para que seja monitorado e avaliado. A precisão dos problemas e necessidades, estabelecimento de possíveis causas dos determinantes e condicionantes da saúde na comunidade, bem como os fatores de risco e vulnerabilidade devem ser constantemente avaliadas.

\section{Definição das ações de extensão a partir do Diagnóstico Comunitário em saúde}

Com a identificação dos problemas, necessidades e recursos, poder-se-á definir o escopo das ações prioritárias reais experimentadas nas comunidades, com estratégias adequadas e avaliação das atividades desenvolvidas para o alcance dos resultados. Propõem-se, aqui, a continuidade do DC pautada em três etapas que garantam o desenvolvimento das ações de extensão, as quais são explanadas sequencialmente. 


\section{Definição de estratégias de Intervenção}

Planejamento que envolve o alcance de metas, organização para ações de intervenção em saúde e definição do quantitativo da população a ser impactada, determinadas a partir do DC, com cronograma, meios, instrumentos e métodos para o desenvolvimento das ações e definição de uso dos recursos identificados no DC devidamente assentados. Nessa etapa, são definidas as modalidades apresentadas pela Resolução № 7, de 18 de Dezembro de 2018: programas, projetos, cursos e oficinas, eventos ou prestação de serviço ${ }^{8}$.

Conceitualmente, o programa é o pilar da extensão composto de atividades e ações que integram os projetos, cursos, eventos e prestação de serviços. É o "guarda-chuva" da extensão universitária, integrado ao ensino e à pesquisa que se volta aos benefícios da sociedade. Os projetos são caracterizados pelo desenvolvimento de ações de extensão a partir de demandas reais identificadas na sociedade, cujos objetivos são programados para alcance em médio e longo prazos, com foco educativo, social, cultural, científico ou tecnológico ${ }^{18-19}$.

Os cursos e oficinas têm como fundamento a capacitação, a atualização, o aperfeiçoamento e o treinamento desenvolvidos de forma teórica ou prática, presencial ou à distância. Os eventos visam a "apresentação e/ou exibição pública, livre ou com clientela específica, do conhecimento ou produto cultural, artístico, esportivo, científico e tecnológico desenvolvido, conservado ou reconhecido pela Universidade" ${ }^{19}$. Pode ser desenvolvido por meio de congresso, seminário, ciclo de debates, exposição, espetáculo, festival, entre outros. A prestação de serviço é desenvolvida por meio da realização de atividades e ações oriundas das demandas sociais, com transferência do conhecimento gerado pela IES. $\mathrm{Na}$ área de saúde, por exemplo, é caracterizada pela assistência ou atendimento prestado. consultorias, assessorias, pesquisas encomendadas, laudos ${ }^{18-19}$.

\section{Execução das Ações}

Aplicação das estratégias de ação conforme a modalidade definida em meio à comunidade, integradas e pactuadas com a equipe e gestores do serviço de saúde, fazendo uso dos recursos disponíveis para o desenvolvimento das ações de intervenção em saúde. Essa etapa é o ponto crucial a ser registrado e gerar indicadores para a construção de relatório da ação de extensão, destacada na Resolução: "As atividades de extensão devem ser sistematizadas e acompanhadas, com o adequado assentamento, além de registradas, fomentadas e avaliadas por instâncias administrativas institucionais, devidamente estabelecidas, em regimento próprio”"

\section{Avaliação dos resultados e autoavaliação}

Possibilita a análise da eficácia e o impacto das ações executadas por meio de informações e dados que permitam avaliar o resultado das ações. A extensão deve estar sujeita à contínua autoavaliação crítica, voltada ao aperfeiçoamento que agregue a formação do estudante, a qualificação do docente, relevância social, a participação dos serviços e a outras dimensões acadêmicas. A avaliação dos resultados da ação desenvolvida e a autoavaliação da extensão, devem incluir a identificação da pertinência da utilização das atividades de extensão na creditação curricular e a demonstração dos resultados alcançados em relação ao público participante ${ }^{8}$.

Nesse processo, destaca-se que o DC favorece o ensino-aprendizagem e a pesquisa, e viabiliza também a extensão curricular universitária, pois atende às exigências determinadas pela Resolução № 7, de 18 de Dezembro de 2018, destacando o cumprimento da formação integral do estudante, cidadão crítico e responsável, diálogo construtivo e transformador com os demais setores da sociedade, respeito e promoção da interculturalidade, promoção de iniciativas que expressem o compromisso social 
com reflexão ética quanto à dimensão social do ensino e da pesquisa, atuação na produção e na construção de conhecimentos atualizados e coerentes com a realidade brasileira ${ }^{8}$.

A construção do DC a fim de programar e desenvolver ações de intervenção em saúde, pautadas na realidade vivida em comunidades, torna o estudante protagonista do conhecimento, no desenvolvimento de habilidades e atitudes baseadas em visão críticareflexiva diante das realidades de saúde vividas pela sociedade. Sugere-se que a aplicação do processo proposto esteja integrado aos componentes disciplinares ou modulares durante a formação acadêmica, constituídos por aulas práticas em meio aos serviços de saúde, principalmente naqueles que contemplem a saúde coletiva.

Assim, esse ensaio reflexivo apresenta as etapas para realização do Diagnóstico Comunitário como proposta de instrumento viável, que favorece e norteia o alcance dos objetivos e diretrizes da curricularização da extensão no ensino universitário em saúde. Acredita-se que a presente discussão contribui para a reflexão de estratégias para implementar a extensão curricular de forma efetiva, cumprindo os quesitos determinados na resolução apresentada.

\section{REFERÊNCIAS}

1. Gonzalez AD, Almeida MJ. Integralidade da saúde: norteando mudanças na graduação dos novos profissionais. Ciênc. saúde coletiva [Internet]. 2010; 15(3):757-762. [Acesso em 15 mar 2021]. Disponível em: http://dx.doi.org/10.1590/S1413-81232010000300018.

2. Almeida MJ. Gestão da escola médica: crítica e autocrítica. Rev. bras. educ. med.[Internet]. 2008; 32(2);202209. [Acesso em 16 mar 2021]. Disponível em: https://doi. org/10.1590/S0100-55022008000200008.

3. Cyrino AP, Godoy D, Cyrino EG. Saúde, ensino e comunidade: reflexões sobre práticas de ensino na atenção primária à saúde. São Paulo: Cultura Acadêmica, 2014.

4. Teixeira CF, Paim JS, Vilas Boas AL. SUS: modelos assistenciais e vigilância da saúde. Inf. Epidemiol. Sus [Internet]. 1998; 7(2):7-28. [Acesso em 18 mar 2021]. Disponível em: http://dx.doi.org/10.5123/S010416731998000200002 .

5. Brandao ERM, Rocha SV, Silva SS. Práticas de integração ensino-serviço-comunidade: Reorientando a formação médica. Rev. bras. educ. med. [Internet]. 2013. 37(4):573577. [Acesso em 18 mar 2021]. Disponível em: https://doi. org/10.1590/S0100-55022013000400013.

6. Moreira COF, Dias MSA. Diretrizes Curriculares na saúde e as mudanças nos modelos de saúde e de educação. ABCS Health Sci. [Internet]. 2015; 40(3):300-305. [Acesso em 25 mar 2021]. Disponível em: https://pesquisa.bvsalud. org/portal/resource/pt/lil-771412

7. Codato LAB. Integração ensino-serviço de saúde: uma compreensão por meio da fenomenologia Heideggeriana [Internet]. [Tese doutorado] Universidade Estadual de Londrina; 2015. [Acesso em 26 mar 2021]. Disponível em: h t t p : / / w w w. bi b li ot e c a digital.uel.br/ document/?code=vtls000200872

8. Brasil. Ministério da Educação. Resolução no 7, DE 18 de dezembro de 2018. [Internet] 2018 [Acesso em 12 mar 2021]. Disponível em: http://portal.mec.gov.br/ index.php?option=com_docman\&view=download\&ali as=104251-rces007-18\&category_slug=dezembro-2018pdf\&Itemid $=30192$

9. Rozin L, Sanches LC, Forte LT, Garbelini MCL. Projetos de extensão das Faculdades Pequeno Príncipe: uma trajetória a compartilhar. Curitiba: CRV; 2020.

10. Egry EY, Fonseca RMGS, Oliveira MAC, Bertolozzi MR. Enfermagem em Saúde Coletiva: reinterpretação da realidade objetiva por meio da ação praxiológica. Rev. Bras. Enferm. [Internet]. 2018; 71(1):710-715. [Acesso em 26 mar 2021]. Disponível em: http://dx.doi.org/10.1590/0034-71672017-0677.

11. Morin E. Os sete saberes necessários à educação do futuro. 2ed. São Paulo: Cortez; Brasília: UNESCO; 2011.

12. Péchevis M. O trabalho do agente comunitário de saúde. Ministério da Saúde. Secretaria de Políticas de Saúde. Departamento de Atenção Básica. Brasília. [Internet]. 2000. [Acesso em 03 abr 2021]. Disponível em: http://bvsms. 
saude.gov.br/bvs/publicacoes/cd09_05c.pdf

13. Becker D, Edmundo K, Nunes NR, Bonatto D, Souza R. Empowerment e avaliação participativa em um programa de desenvolvimento local e promoção da saúde. Ciênc. saúde coletiva. [Internet]. 2004; 9(3):655-667. [Acesso em 10 abr 2021]. Disponível em:https://doi.org/10.1590/S141381232004000300017.

14. Duncan BB, Schimidt MI, Giugliani ERJ. Medicina ambulatorial: condutas de atenção primária baseada em evidências. 3ed. Porto Alegre: Artmed; 2004.

15. Tomasi YT, Souza JB, Madureira VSF. Diagnóstico comunitário na Estratégia Saúde da Família: potencialidades e desafios. Ver enferm UFPE online. [Internet]. 2018; 12(6):1546-53. [Acesso em 10 abr 2021]. Disponível em:https://periodicos.ufpe.br/revistas/revistaenfermagem/ article/view/230505/29180
16. Dominguz BNR. O programa saúde da família: como fazer. São Paulo: Milenium; 1998.

17. Mauro MYC. Diagnóstico de saúde de comunidade com atividade discente de enfermagem, utilizando o método de projeto. Rev bras enferm. [Internet]. 1987; 40(2-3):126131. [Acesso em 15 abr 2021]. Disponível em: http://dx.doi. org/10.1590/S0034-71671987000300009

18. Oliveira F, Goulart PM. Fases e faces da extensão universitária: rotas e concepções. Rev. Ciênc. [Internet]. 2015; 11(3): 8-2. [Acesso em 25 abr 2021]. Disponível em: https://ojs.unesp.br/index.php/revista_proex/article/ view/1225/1165

19. FORPROEX. Plano Nacional de Extensão universitária: versão final. 2001. [Acesso em25 abr 2021]. Disponível em:http://www.renex.org.br/documentos/EncontroNacional/1995-IX-Encontro-Nacionaldo-FORPROEX.pdf 